\title{
Evaluating the Possibility of Defining Cut-Off Points for $\triangle F A \%$ in Order to Differentiate Four Major Types of Peri-Tumoral White Matter Tract Involvement
}

\author{
Tourisa Deilami, ${ }^{1,}$ Homayoun Hadizadeh Kharrazi, ${ }^{1}$ Amir Saied Seddighi, ${ }^{2}$ Parin Tanzifi, \\ Reza Tayebivaljouzi, ${ }^{4}$ Fatemeh Zamani, ${ }^{1}$ and Atefeh Chavoshzadeh Tafti ${ }^{5}$ \\ ${ }_{2}^{1}$ Department of Radiology, Tehran University of Medical Sciences, Tehran, Iran \\ ${ }_{3}^{2}$ Department of Neurosurgery, Shahid Beheshti University of Medical Sciences, Tehran, Iran \\ ${ }^{3}$ Department of Pathology, Tehran University of Medical Sciences, Tehran, Iran \\ ${ }^{4}$ Department of Radiology, Shahid Beheshti University of Medical Sciences, Tehran, Iran \\ ${ }^{5}$ Department of Neurology, Tehran University of Medical Sciences, Tehran, Iran \\ *Corresponding author: Tourisa Deilami, Department of Radiology, Tehran University of Medical Sciences, Tehran, Iran. Tel: +98-2188001012, E-mail: tourisadeilami@gmail.com
}

Received: December 9, 2012; Revised: November 4, 2013; Accepted: January 13, 2014

\begin{abstract}
Background: Diffusion tensor imaging (DTI) and its different scalar values such as fractional anisotropy (FA) have recently been used for evaluation of peri-tumoral white matter (WM) involvement to help define safer surgical excision margins.

Objectives: The purpose of this study is to evaluate the possibility of defining diagnostic cut-off points for differentiating four major types of peri-tumoral WM involvement using FA.

Patients and Methods: DTI was performed in 12 patients with high presumption of having brain tumors, on a 1.5 T MRI scanner. DTI data was processed by MedINRIA software. Two-hundred region of interests (ROI) were evaluated: 100 in the lesion zone and the rest in the normal WM in the contralateral hemisphere. FA value related to each ROI was measured, and the percentage of FA decrement $(\Delta \mathrm{FAs} \%)$ was calculated.

Results: Of the 100 ROIs on the lesion side, 74 were related to high-grade lesions, 23 to low-grade ones, and three to "gliosis". There were 54 "infiltrated", 22 "displaced", 15 "disrupted", and 9 "edematous" tracts. The major type of fiber involvement, both in low-grade and high-grade tumors was "infiltrated, whereas "edematous" fibers comprised the minority. $\Delta \mathrm{FA} \%$ was more than - 35 for "displaced" and "edematous" fibers, and less than -35 for the majority of "disrupted" ones, but "infiltrated" fibers had scattered distribution. Mean $\triangle \mathrm{FA} \%$ was the least for "disrupted", followed by "infiltrated", "edematous" and "displaced" parts.

Conclusion: Introducing definite diagnostic cut-points was not possible, due to overlap. Based on the fact that "disruption" is the most aggressive process, a sensitivity analysis was carried out for "disrupted" fibers for several presumptive cut-off points.
\end{abstract}

Keywords: Diffusion Tensor Imaging; Brain Neoplasms; White Matter; Anisotropy

\section{Background}

Non-invasive mapping of central nervous system (CNS) microstructure has been recently developed by magnetic resonance imaging (MRI) diffuse tensor imaging (DTI). The technique is based on Brownian motion of water molecules. Complex tissue barriers such as cell membrane, myelin sheath or even pathological tissue barriers can alter this molecular movement by hindrance. The directional variation of water molecules is termed diffusion anisotropy that facilitates the visualization of major fiber tracts, as diffusion component parallel to the tract direction is larger than its perpendicular counterpart $(1,2)$. Different scalar values defined for DTI, such as fractional anisotropy (FA) have been used for evaluation of white matter (WM) involved by the tumoral process. FA varies from 0 to 1 , where the lower and upper limits represent isotropic diffusion and directional preference along the major eigenvector, respectively (3-5).
WM tract involvement due to tumor extension is classified as infiltrated, displaced, disrupted, and edematous (4, 6). Recent efforts have been based on distinguishing these types of lesions using DTI and its metrics such as FA (6-13)

\section{Objectives}

In the current study, we assessed the value of FA for differentiating these types of WM tract involvement.

\section{Patients and Methods}

In a case-series study, 12 patients with a high presumption of having tumoral brain lesions were recruited for investigation in Babak imaging center in 2011, neither of whom having additional intracranial lesions, history of seizure or anti-epileptic medications. The ethics committee of Tehran University of Medical Sciences (TUMS) approved the study protocol.

Copyright (C) 2015, Tehran University of Medical Sciences and Iranian Society of Radiology. This is an open-access article distributed under the terms of the Creative Commons Attribution-NonCommercial 4.0 International License (http://creativecommons.org/licenses/by-nc/4.0/) which permits copy and redistribute the material just in noncommercial usages, provided the original work is properly cited. 
A1.5T MRI scanner (Siemens, Magnetom Avanto, Germany) with 18 channels, max gradient strength $=45 \mathrm{mT} / \mathrm{m}$, slew rate $=200 \mathrm{~T} / \mathrm{m} / \mathrm{s}$ and a head coil with 8 channels was used. The images were obtained by application of a diffusion-weighted echo planar pulse sequence (SSEPI). High resolution structural images were acquired using a 3D T1-Weighted gradient echo sequence. All raw data were processed by MedINRIA software (version1.9.0) with the same setting.

The obtained scalar values were based on a ROI analysis technique, using color-coded FA maps in the peritumoral (PT) edema and some abnormally appearing WM tracts located in the PT region not within edema. The tracts in the contralateral hemisphere were employed as reference for normalization. In most cases, more than one region of interest (ROI) was selected to acquire more accurate scalar values from different regions of the same WM tract.

We categorized WM tract involvement into four types according to the study conducted by Witwer et al. (14). In two cases, however, we encountered fiber tracts that could not be placed in any of Witwer et al."s categories. The abnormal fibers had increased volume and also increased FA in comparison with their corresponding fibers. With recourse to the literature, we finally decided to classify them as "infiltrated"(15).

Since all ROIs were defined by a hand-drawn procedure, a single specialist was employed only for this part of the work to minimize inter-observer errors. The ROIs were defined on the lesion side prior to the normal side and drawn symmetrically based on and almost equal to the size and shape of the ROIs on the lesion side.

For more accurate drawing of ROIs, the atlas provided by Kenichi et al. (16) was used in all steps. The formula proposed by Yen et al. for percentage of FA decrement $(\triangle \mathrm{FA} \%)(4)$ was used. This is defined as the percentage of FA decrement in the tumor to the normal FA in the corresponding tract (Equation 1)

$$
\Delta \mathrm{FA} \%=\frac{\mathrm{FA}_{\text {lesion }}-\mathrm{FA}_{\text {normal }}}{\mathrm{FA}_{\text {normal }}} \times 100
$$

Applying FA normal as the denominator eliminates the discrepancies between different individuals and different WM tracts. In this study, the standard deviation (SD) of normal FAs was considered as a benchmark for the classification of FAs into normal or abnormal.

The results were reported as Mean \pm SD for the quantitative variables and percentages for the categorical variables. P values of $\leq 0.05$ were considered statistically significant. All statistical analyses were carried out using SPSS version 16.0 (SPSS Inc., Chicago, IL, USA).

\section{Results}

The imaging procedure was done prior to the surgical biopsy on 12 patients. According to the proven pathology, 7 patients had high grade tumors (grade III, IV), four had low grade lesions, and finally one had gliosis.

Overall, 200 ROIs were evaluated: 100 in the lesion zone and 100 in the contralateral normal WM and the FA was measured, based on which the percentage of FA was calculated.

Of the 100 ROIs on the lesion side, 74 were from highgrade lesions, 23 from low-grade ones, and three were related to the gliosis. We applied the SPSS software to divide the percentage of FA values into four quartiles (Table 1). The distribution of displaced and edematous lesions were mostly in the third and fourth quartiles (more than -35) and disrupted lesions were mainly in the first three quartiles (less than 0 ).

$\triangle \mathrm{FA} \%$ ranges are presented in Table 2 . As noted, the least mean $\triangle \mathrm{FA} \%$ can be seen in "disrupted" lesions (-63.52) and the most for "displacement". In infiltrated lesions, $\triangle F A \%$ values were significantly higher outside the PT edema than within the edema, but such significant difference was not found in displaced fibers. It was also revealed that mean $\triangle \mathrm{FA} \%$ of ROIs located within the PT edema was significantly lower in comparison with the fibers outside the edema and mean $\triangle F A s \%$ within and outside the edema lie in the second and third quartiles, respectively. The relationship between the WHO grading of the tumors and different types of fiber involvement is demonstrated in Table 3. It shows that the major type of involvement, both in low-grade and high-grade tumors, is infiltration, whereas edematous fibers comprise the minority.

\begin{tabular}{|c|c|c|c|c|}
\hline \multirow{2}{*}{ Type of Involvement } & \multicolumn{4}{|c|}{$\Delta$ FA\% Strata } \\
\hline & 1st Quartile (<-60) & 2nd Quartile (-60 to -35$)$ & 3rd Quartile (-34.9 to 0$)$ & 4th Quartile $(>0)$ \\
\hline Infiltration & $14(25.9)$ & $20(37.0)$ & $18(33.3)$ & $2(3.7)$ \\
\hline Within Edema & $14(34.1)$ & $19(46.3)$ & $7(17.1)$ & $1(2.4)$ \\
\hline Outside Edema & 0 & $1(7.7)$ & $11(84.6)$ & $1(7.7)$ \\
\hline Displacement & 0 & 0 & $7(31.8)$ & $15(68.2)$ \\
\hline Within Edema & 0 & 0 & 0 & $1(100)$ \\
\hline Outside Edema & 0 & 0 & $7(33.0)$ & $14(66.7)$ \\
\hline Disruption & $9(60.0)$ & $5(33.3)$ & $1(6.7)$ & $0(0.0)$ \\
\hline Within Edema & $9(60.0)$ & $5(33.3)$ & $1(6.7)$ & 0 \\
\hline Outside Edema & 0 & 0 & 0 & 0 \\
\hline Edema & 0 & 0 & $5(55.6)$ & $4(44.4)$ \\
\hline
\end{tabular}

${ }^{\mathrm{a}}$ Data are presented as No. (\%); $\triangle \mathrm{FA} \%$ : Percentage of Fractional Anisotropy Decrement. 
Deilami Tet al.

\begin{tabular}{|c|c|c|c|}
\hline Involvement & Mean $\Delta \mathrm{FA}, \%$ & FA Range, Lesion & $\Delta$ FA Range, $\%$ \\
\hline \multicolumn{4}{|l|}{ Displacement } \\
\hline Total & $4.564^{\mathrm{a}}$ & $0.39-0.87$ & $(-11.44)-(+18.41)$ \\
\hline Within edema & $17.08^{b}$ & $0.67-0.67$ & $(+17.08)-(+17.08)$ \\
\hline Outside edema & $3.97^{\mathrm{C}}$ & $0.39-0.87$ & $(-11.44)-(+18.41)$ \\
\hline PValue & NS & & \\
\hline \multicolumn{4}{|l|}{ Disruption } \\
\hline Total & $-63.52^{a}$ & $0.09-0.43$ & $(-83.75)-(-21.39)$ \\
\hline Within edema & $-63.52^{a}$ & $0.09-0.43$ & $(-83.75)-(-21.39)$ \\
\hline \multicolumn{4}{|l|}{ Infiltration } \\
\hline Total & $-42.70^{a}$ & $0.09-0.77$ & $(-83.65)-(+8.47)$ \\
\hline Within edema & $-50.62^{b}$ & $0.05-0.65$ & $(-83.65)-(+1.09)$ \\
\hline Outside edema & $-17.72^{\mathrm{C}}$ & $0.31-0.77$ & $(-37.46)-(+8.47)$ \\
\hline PValue & 0.021 & & \\
\hline Edema & $3.98^{\mathrm{a}}$ & $0.35-0.76$ & $(-15.36)-(+38.41)$ \\
\hline
\end{tabular}

Table 3. Relationship Between Tumor Grade and Type of White Matter Involvement ${ }^{\mathrm{a}}$

\begin{tabular}{|lc}
\hline Grading & Values \\
\hline Low Grade Tumors & \\
\hline Displacement & $7(30.5)$ \\
\hline Infiltration & $15(65.2)$ \\
\hline Disruption & $1(4.3)$ \\
\hline Edema & $0(0.0)$ \\
\hline High Grade Tumors & \\
\hline Edema & $9(12.2)$ \\
\hline Disruption & $14(19)$ \\
\hline Infiltration & $36(48.6)$ \\
\hline Displacement & $15(20.2)$ \\
\hline a Data are presented as No. (\%).
\end{tabular}

\section{Discussion}

In this study, we applied DTI and FA value for categorization of fiber involvement inside and outside PT edema. In neither "displaced" nor "edematous" fibers, $\triangle \mathrm{FA} \%$ was less than -35. Furthermore, none of the "disrupted" fibers were found to have a positive $\Delta \mathrm{FA} \%$, and actually their distribution was mostly in the first and second quartiles. Therefore, it can be concluded that most "disrupted" fibers possess a $\Delta \mathrm{FA} \%$ of less than -35. Whereas, the "infiltrated" fibers had scattered distribution among all quartiles (mostly in negative quartiles), so we were unable to infer that all fibers with $\Delta \mathrm{FA} \%$ of less than -35 can be categorized as "disruption". Similarly, there is overlap in the third and fourth quartiles between "edematous" and "displaced" fibers making it impossible to establish a definite cut-off point for them.

The majority of the "displaced" fibers located outside the PT edema possessed a positive $\Delta \mathrm{FA} \%$ and thus were placed in the fourth quartile. Although Yen et al. (4) had come to the conclusion that $\triangle \mathrm{FA} \%$ of less than -30 is consistent mostly with "disruption", this cut-off value was not reached in our study. Instead, $\Delta \mathrm{FA} \%$ less than -35 can be suggestive of either "infiltration" or "disruption" that cannot be subdivided.

In addition, as no "disrupted" fiber was found in the fourth quartile with positive $\triangle \mathrm{FA} \%$, we deduced that a positive $\triangle \mathrm{FA} \%$ can rule out the diagnosis of "disruption". When $\triangle F A \%$ is more (positive) than -35 , distinction between the types of fiber involvement is elusive due to their overlap.

Data analysis in Table 2 reveals two points. First, clear determination of fiber involvement cannot be done using $\triangle \mathrm{FA} \%$. Moreover, mean $\triangle \mathrm{FA} \%$ for four types of involvement, is the least for "disruption"(-63.52) and gradually increases toward "displacement" (4.58) with "infiltration" and "edematous involvement" in between ( $P$ value $=0.002$ ). This correlates well with the fact that "disruption" is overall the most aggressive process and the aggressiveness gradually decreases toward displacement, in such a way that more destructive patterns, i.e. "disruption" and "infiltration" have negative mean $\triangle \mathrm{FAs} \%$, but less aggressive types of involvement have positive mean $\triangle \mathrm{FAs} \%$.

Compared to the findings of Yen et al. we classified all lesion involvement types into two categories according to their location inside or outside the PT edema (4). This does not apply to edematous fibers, which are all located within the edema. 
In the "infiltration" subgroup, we detected that the mean $\triangle \mathrm{FA} \%$ outside the PT edema is significantly larger than the mean $\triangle F A \%$ within the edema (P value $<0.001$ ).

The most common involvement here, both in high-grade and low-grade lesions, was "infiltration". This contrasts the results of the study conducted by Li et al. (1), in which "infiltration" and "destruction" were the most common involvement in high-grade tumors, and "displacement" was the major involvement in low-grade ones.

Although this study does not yield definite diagnostic cut-off points for $\triangle \mathrm{FAs} \%$ between four major types of WM tract involvement, a sensitivity analysis, which includes sensitivity, specificity, positive and negative predictive values, and $\mathrm{P}$ values for each presumptive cut-off is performed for "disrupted" fibers (Table 4). Three examples of DTI reconstruction are shown in Figures 1-3.

\begin{tabular}{|c|c|c|c|c|c|c|c|c|}
\hline $\begin{array}{l}\text { Presumptive } \triangle F A \% \\
\text { Cut Points }\end{array}$ & $\begin{array}{c}\text { With } \\
\text { Disruption }\end{array}$ & $\begin{array}{l}\text { Without } \\
\text { Disruption }\end{array}$ & Sensitivity & Specificity & PPV & NPV & PValue & $\begin{array}{c}\text { Odds ratio } \\
\text { (95\% Confidence Interval) }\end{array}$ \\
\hline$\Delta$ FA $\%$ cut point: -10 & & & 100 & 38.8 & 22.4 & 100 & 0.003 & \\
\hline$<-10$ & 15 & 52 & & & & & & \\
\hline$\geq-10$ & 0 & 33 & & & & & & \\
\hline$\Delta$ FA $\%$ cut point:-30 & & & 93.3 & 54.1 & 26.4 & 97.9 & $<0.001$ & $16.5(2.07-131.27)$ \\
\hline$<-30$ & 14 & 39 & & & & & & \\
\hline$\geq-30$ & 1 & 46 & & & & & & \\
\hline$\Delta$ FA\% cut point: - 60 & & & 60 & 83.5 & 39.1 & 92.2 & $<0.001$ & $7.6(2.33-24.79)$ \\
\hline$<-60$ & 9 & 14 & & & & & & \\
\hline$\geq-60$ & 6 & 71 & & & & & & \\
\hline$\Delta$ FA\% cut point: -65 & & & 60 & 84.7 & 40.9 & 92.3 & $<0.001$ & $8.30(2.52-27.3)$ \\
\hline$<-65$ & 9 & 13 & & & & & & \\
\hline$\geq-65$ & 6 & 72 & & & & & & \\
\hline$\Delta$ FA\% cut point:-70 & & & 53.3 & 92.9 & 57.1 & 91.9 & $<0.001$ & $15(4.05-55.8)$ \\
\hline$<-70$ & 8 & 6 & & & & & & \\
\hline$\geq-70$ & 7 & 79 & & & & & & \\
\hline$\Delta$ FA\% cut point: -80 & & & 6.7 & 97.6 & 33.3 & 85.6 & 0.367 & $2.96(0.25-34.91)$ \\
\hline$<-80$ & 1 & 2 & & & & & & \\
\hline$\geq \mathbf{8 0}$ & 14 & 83 & & & & & & \\
\hline
\end{tabular}

a Abbreviations: NPV, negative predictive value; PPV, positive predictive value.

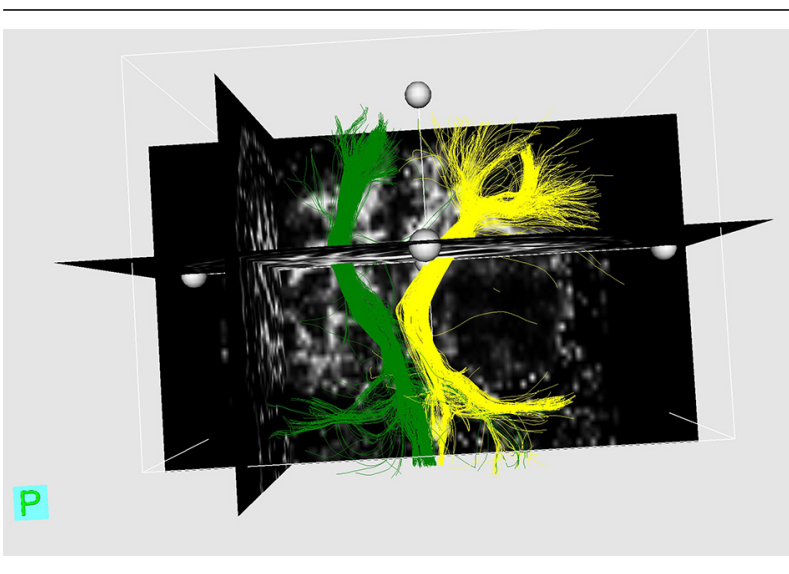

Figure 1. DTI reconstruction, the normal corticospinal tract (green) versus the contralateral (yellow) tract, displaced by the tumor bulk, viewed from the posterior

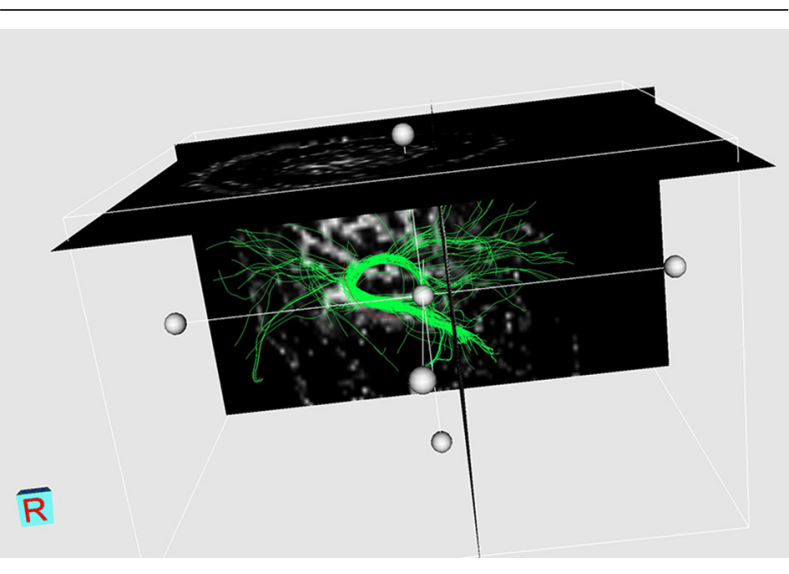

Figure 2. DTI reconstruction, the normal fornix (green) versus the contralateral fornix (purple), which is mostly destroyed by the tumor bulk (not shown). Reconstruction schematically shows the disrupted fornix as just one single remained normal white matter fiber 


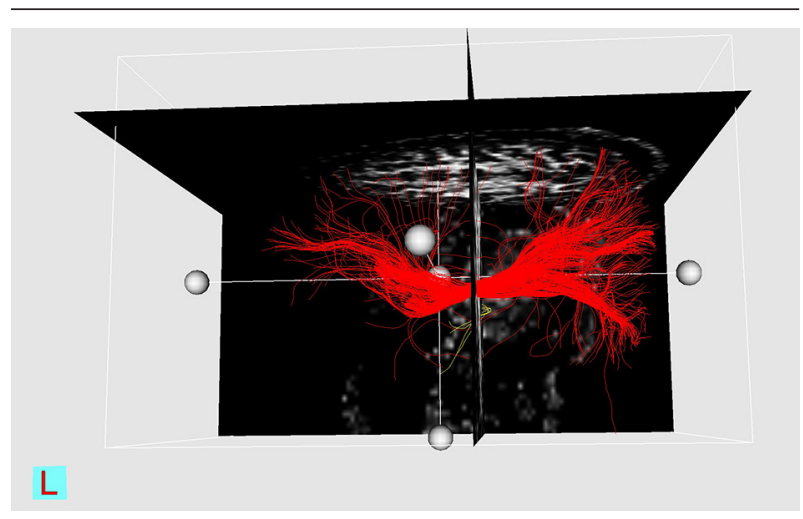

Figure 3. DTI reconstruction, the normal IFO (inferior fronto-occipital fasciculus) (red) versus the contralateral (yellow) side destroyed by the tumor bulk, viewed from lateral. Reconstruction schematically shows the disrupted tract as having a few maintained white matter fibers

\section{Financial Disclosure}

Authors have no financial interests related to the material in the manuscript.

\section{Funding/Support}

The research was supported financially by the authors themselves, not any other organization.

\section{Authors' Contributions}

Study conception and design: Tourisa Deilami, Homayoun Hadizadeh Kharrazi, Amir Saied Seddighi, and Atefeh Chavoshzadeh Tafti; Acquisition of data: Tourisa Deilami and Homayoun Hadizadeh Kharrazi; Analysis and interpretation of data: Tourisa Deilami, Homayoun Hadizadeh Kharrazi, and Reza Tayebivaljouzi; Drafting of manuscript: Tourisa Deilami, Reza Tayebivaljouzi, Amir Saied Seddighi, Parin Tanzifi, and Fatemeh Zamani; Critical revision: Tourisa Deilami and Homayoun Hadizadeh Kharrazi; Statistical analysis: Parin Tanzifi; Providing the textbooks, materials, and the articles: Tourisa Deilami and Homayoun Hadizadeh Kharrazi.

\section{References}

1. Li X, Yu RT, Xu K, Li FC, Fan YC, Gao WC, et al. [Application of dif- fusion tensor imaging in preoperation and postoperation patients of glioma with 3.0 Tesla MRI]. Zhonghua Yi Xue Za Zhi. 2009;89(19):1300-4.

2. Li YZ, Huang ZL, Wei DN, Xie CM, He HQ, Wei YF, et al. [Diffusion tensor imaging of the white matter tracts in preoperative patients with cerebral neoplasm]. Nan Fang Yi Ke Da Xue Xue Bao. 2006;26(11):1648-51.

3. Inoue T, Ogasawara K, Beppu T, Ogawa A, Kabasawa H. Diffusion tensor imaging for preoperative evaluation of tumor grade in gliomas. Clin Neurol Neurosurg. 2005;107(3):174-80.

4. Yen PS, Teo BT, Chiu CH, Chen SC, Chiu TL, Su CF. White Matter tract involvement in brain tumors: a diffusion tensor imaging analysis. Surg Neurol. 2009;72(5):464-9.

5. Sinha S, Bastin ME, Whittle IR, Wardlaw JM. Diffusion tensor MR imaging of high-grade cerebral gliomas. AJNR Am J Neuroradiol. 2002;23(4):520-7.

6. Provenzale JM, McGraw P, Mhatre P, Guo AC, Delong D. Peritumoral brain regions in gliomas and meningiomas: investigation with isotropic diffusion-weighted MR imaging and diffusiontensor MR imaging. Radiology. 2004;232(2):451-60.

7. Beppu T, Inoue T, Shibata Y, Kurose A, Arai H, Ogasawara K, et al. Measurement of fractional anisotropy using diffusion tensor MRI in supratentorial astrocytic tumors. J Neurooncol. 2003;63(2):109-16.

8. Beppu T, Inoue T, Shibata Y, Yamada N, Kurose A, Ogasawara K, et al. Fractional anisotropy value by diffusion tensor magnetic resonance imaging as a predictor of cell density and proliferation activity of glioblastomas. Surg Neurol. 2005;63(1):56-61.

9. Price SJ, Jena R, Burnet NG, Hutchinson PJ, Dean AF, Pena A, et al. Improved delineation of glioma margins and regions of infiltration with the use of diffusion tensor imaging: an image-guided biopsy study. AJNR Am J Neuroradiol. 2006;27(9):1969-74.

10. Hayashida Y, Hirai T, Morishita S, Kitajima M, Murakami R, Korogi Y, et al. Diffusion-weighted imaging of metastatic brain tumors: comparison with histologic type and tumor cellularity. AJNR Am J Neuroradiol. 2006;27(7):1419-25.

11. Jenkinson MD, Du Plessis DG, Walker C, Smith TS. Advanced MRI in the management of adult gliomas. Br J Neurosurg. 2007;21(6):550-61.

12. Tsuchiya K, Fujikawa A, Nakajima M, Honya K. Differentiation between solitary brain metastasis and high-grade glioma by diffusion tensor imaging. Br J Radiol. 2005;78(930):533-7.

13. Deng Z, Yan Y, Zhong D, Yang G, Tang W, Lu F, et al. Quantitative analysis of glioma cell invasion by diffusion tensor imaging. $J$ Clin Neurosci. 2010;17(12):1530-6.

14. Witwer BP, Moftakhar R, Hasan KM, Deshmukh P, Haughton V, Field A, et al. Diffusion-tensor imaging of white matter tracts in patients with cerebral neoplasm. J Neurosurg. 2002;97(3):568-75.

15. Helton KJ, Phillips NS, Khan RB, Boop FA, Sanford RA, Zou P, et al. Diffusion tensor imaging of tract involvement in children with pontine tumors. AJNR Am J Neuroradiol. 2006;27(4):786-93.

16. Kenichi O, Anderia F, Peter CM van Z, Susumu M. MRI atlas of human white matter. London: 2011. 